\title{
Silencing Notch-1 induces apoptosis and increases the chemosensitivity of prostate cancer cells to docetaxel through Bcl-2 and Bax
}

\author{
QI-FA YE ${ }^{1}$, YI-CHUAN ZHANG ${ }^{1,2}$, XIAO-QING PENG ${ }^{3}$, ZHI LONG ${ }^{2}$, YING-ZI MING ${ }^{1}$ and LE-YE HE ${ }^{2}$ \\ ${ }^{1}$ Engineering and Technology Research Center for Transplantation Medicine of the National Ministry of Health; \\ Departments of ${ }^{2}$ Urology, and ${ }^{3}$ Gastroenterology, The Third Xiangya Hospital, \\ Central South University, Changsha, Hunan 410013, P.R. China
}

Received October 20, 2011; Accepted December 29, 2011

DOI: $10.3892 / \mathrm{ol} .2012 .572$

\begin{abstract}
Although docetaxel-based chemotherapy is therapeutically efficacious, drug resistance often leads to treatment failure in castration-resistant prostate cancer patients. The Notch signaling pathway plays a key role in prostate development and prostate cancer. We investigated whether silencing Notch-1 has therapeutic potential for the treatment of prostate cancer. To determine this, we performed cell and molecular analyses following the silencing of the Notch-1 gene in PC-3 castration-resistant prostate cancer cells using small interfering RNA. The results demonstrated that silencing the Notch-1 gene effectively inhibits proliferation and induces apoptosis in PC-3 cells. In addition, docetaxel treatment results in decreased proliferation and increased apoptosis in the Notch-1-silenced cells compared to the control PC-3 cells. Docetaxel treatment was also accompanied by an upregulation of Bax and a downregulation of Bcl-2. Thus, Notch-1 silencing downregulates the anti-apoptotic protein $\mathrm{Bcl}-2$, and upregulates the pro-apoptotic protein Bax, which ultimately results in increased sensitivity of PC-3 cells to docetaxel. Taken together, these results suggest that Notch-1 is potentially an effective target for treating castration-resistant prostate cancer.
\end{abstract}

\section{Introduction}

According to recent data from the American Cancer Society, prostate cancer $(\mathrm{PCa})$ has the highest incidence rate and the second highest mortality rate among males in the United States of America (1). Most progressive PCa patients who receive hormone treatment exhibit tumor progression and require chemotherapy within 18-24 months (2), at which

Correspondence to: Dr Le-Ye He, Department of Urology, The Third Xiangya Hospital, Central South University, Changsha, Hunan 410013, P.R. China

E-mail: leyehe@csu.edu.cn

Key words: Notch signaling, prostate cancer, small interfering RNA, apoptosis, Bcl-2, Bax point their condition is termed castration-resistant prostate cancer (CRPC), also known as androgen-independent prostate cancer. One chemotherapy regimen for CRPC currently recommended by several authoritative guidelines, including the National Comprehensive Cancer Network (NCCN) and the European Association of Urology (EAU) $(3,4)$, is a combined docetaxel and prednisone therapy. Despite the efficacy of docetaxel-based chemotherapy, tumor progression occurs following a median of 6.3 months, resulting in treatment failure (5). The Food and Drug Administration (FDA) has not yet approved an alternative drug therapy for PCa patients in whom docetaxel therapy is unsuccessful. Therefore, current studies regarding the ability to reverse drug resistance and enhance sensitivity to docetaxel chemotherapy are ongoing.

The Notch family is an evolutionarily conserved family of transmembrane receptor proteins. The Notch signaling pathway plays a key role in cell differentiation, proliferation, survival and apoptosis, and is thus significant for the development and normal function of a number of organs. In mammals, four Notch receptors (Notch-1-4) and five ligands (Dll-1,3 and 4 and Jagged-1 and 2) have been identified (6). All homologous Notch receptors contain a Notch intracellular domain (NICD), an extracellular domain and a transmembrane domain. The Notch signaling pathway is activated when the Notch ligand, adjacent to the cell surface, interacts with the NICD. In response, the Notch receptor then undergoes cleavage by the TNF- $\alpha$-converting enzyme (TACE) and the $\gamma$-secretase/presenilin complex, releasing the NICD. The NICD, once separated from the cell membrane, translocates into the cell nucleus and interacts with the CSL protein $[\mathrm{CBF} 1 / \mathrm{Su}(\mathrm{H}) / \mathrm{Lag} 1]$ to activate transcription of the Notch target genes (7). There are several confirmed Notch target genes, including hairy enhancer of split (Hes) and the NF- $\kappa \mathrm{B}$ family members, Hey, cyclin-D1 and P21 $(8,9)$.

The Notch signaling pathway is significant in the early development of the prostate $(10,11)$, and high levels of Notch receptor expression, particularly Notch-1, are associated with PCa. Notch-1 is highly expressed in several PCa cell lines, including the androgen-independent DU145, PC-3, 22Rr1 and $\mathrm{C} 4-2 \mathrm{~B}$ cell lines, and the androgen-dependent LNCaP cell line (12-15). Notch-1 has also been demonstrated to be 
overexpressed in animal models of prostate cancer $(12,16)$ and in human PCa samples (14). Several studies have revealed that inhibition of Notch-1 expression increases apoptosis and inhibits the proliferation and invasiveness of tumor cells; effects that are associated with the Akt, mTOR, NF- $\kappa \mathrm{B}$, MMP-9 and uPA signaling pathways $(14,15)$. Therefore, we explored the therapeutic potential of inhibiting Notch signaling in the treatment of CRPC.

We used small interfering RNA (siRNA) against Notch-1 to inhibit Notch-1 signaling in PC-3 cells and to determine the effects of silencing Notch-1 on docetaxel sensitivity. Our results demonstrated that a decreased Notch-1 expression leads to decreased proliferation and increased apoptosis of tumor cells. In addition, we found that silencing Notch-1 significantly increases the sensitivity of PC-3 cells to docetaxel chemotherapy; an effect that appears to result from the regulation of the expression of Bcl-2 family members by the Notch signaling pathway. Overall, our results suggest that Notch-1 is an effective potential target for CRPC treatment.

\section{Materials and methods}

Cell culture. The PC-3 cell line was originally obtained from the American Type Culture Collection. Cells were cultured in RPMI-1640 medium supplemented with 10\% FBS and $100 \mu \mathrm{g} / \mathrm{ml}$ kanamycin (Sigma, St. Louis, MO, USA) at $37^{\circ} \mathrm{C}$ in a $5 \% \mathrm{CO}_{2}$ humidified incubator.

The study was approved by the ethics committee of the Third Xiangya Hospital of Central South University, Changsha, China.

Reagents. Docetaxel was purchased from Sanofi-Aventis (Dagenham, UK). The Opti-MEM ${ }^{\circledR}$ I serum-free culture medium and the Lipofectamine ${ }^{\mathrm{TM}} 2000$, used for transfection, and the Total RNA Extraction kit were purchased from Invitrogen Life Technologies (Carlsbad, CA, USA). The MTT Cell Proliferation and Cytotoxicity Assay kit and the Annexin V-FITC and PI Apoptosis Detection kit were purchased from KeyGen Biotech (Nanjing, China). The SYBR-Green PCR Master mix was purchased from Fermentas (Burlington, ON, Canada). The Total Protein Extraction kit was purchased from ProMab (Changsha, China). The rabbit antibodies against Notch-1, Bcl-2 and Bax, and the mouse antibody against GAPDH were purchased from Santa Cruz Biotechnology Inc. (Santa Cruz, CA, USA).

Notch-1 siRNA transfection. Three different siRNAs targeting specific sequences of Notch-1 (NM_017617) and a negative control scrambled siRNA, which was not homologous to any gene, were synthesized by GenePharma (Shanghai, China). The sequences of the three Notch-1 siRNAs were as follows: i) Notch-1 siRNA-2010: sense, 5'-CAGGGAGCA UGUGUAACAUTT-3' and antisense, 5'-AUGUUA CACAUGCUCCCUGTT-3'; ii) Notch-1 siRNA-6150: sense, 5'-GGGCUAACAAAGAUAUGCATT-3' and antisense, 5'-UGCAUAUCUUUGUUAGCCCTT-3'; iii) Notch-1 siRNA-780: sense, 5'-GUCCAGGAAACAACUGCAATT-3' and antisense, 5'-UUGCAGUUGUUUCCUGGACTT-3'. The PC-3 cells were seeded in 6 -well plates $\left(4-5 \times 10^{4}\right.$ cells/well $)$ and cultured in $2 \mathrm{ml}$ basic culture medium containing $10 \%$
FBS overnight until the cells were $70 \%$ confluent. The siRNA-Lipofectamine 2000 complex was pre-mixed according to the manufacturer's instructions and added to the 6-well plates. To select the most effective siRNA sequence, we analyzed Notch-1 expression in the cells using real-time PCR and western blot analysis $48 \mathrm{~h}$ following transfection.

Groups. The five groups of PC-3 cells were treated differently in this study: i) Control: PC-3 cells; ii) Scrambled: scrambled siRNA-transfected PC-3 cells; iii) NS: selected Notch-1 siRNA-transfected PC-3 cells; iv) Doce: PC-3 cells exposed to $58 \mathrm{~nm}$ docetaxel; v) NS + Doce: $48 \mathrm{~h}$ following transfection with Notch-1 siRNA, cells were treated with $58 \mathrm{~nm}$ docetaxel.

Detection of inhibition of cell growth using MTT assays. The PC-3 cells in the logarithmic phase were seeded in 96-well plates. The cells were transfected with Notch-1 siRNA or scrambled siRNA. Forty-eight hours following transfection with Notch-1 siRNA, cells were treated with $58 \mathrm{~nm}$ docetaxel. A total of $24 \mathrm{~h}$ later, $50 \mu \mathrm{l}$ of a $5 \mathrm{mg} / \mathrm{ml}$ MTT solution was added to each well and then incubated for $4 \mathrm{~h}$. The reaction was terminated by adding $150 \mu \mathrm{l}$ DMSO, and the optical density (OD) of each well was measured using a microplate reader (Bio-Rad, Hercules, CA, USA) at a detection wavelength of $570 \mathrm{~nm}$. The experiment was performed in triplicate, and the survival rate was calculated by subtracting the background OD value (complete culture medium without cells) from the OD value from each test well.

Detection of cell apoptosis using flow cytometry. A total of $48 \mathrm{~h}$ following siRNA transfection, the cells were digested, centrifuged and washed with pre-cooled PBS. Cells were then washed with binding buffer containing $10 \mathrm{mmol} / \mathrm{l} \mathrm{HEPES}$, $140 \mathrm{mmol} / \mathrm{l} \mathrm{NaCl}$ and $2.5 \mathrm{mmol} / \mathrm{l} \mathrm{CaCl}_{2}$ at $\mathrm{pH} 7.4$ and then re-suspended. Each sample $\left(10^{5}-10^{6}\right.$ cells $)$ was treated with $5 \mu \mathrm{l}$ of Annexin V-FITC and $10 \mu \mathrm{l}$ propidium iodide (PI), mixed, and then incubated in the dark at room temperature for $15 \mathrm{~min}$. Fluorescence was detected using a flow cytometer (FACSAria, BD Bioscience) within $1 \mathrm{~h}$. The results were averaged and the percentage of apoptosis was calculated using the CellQuest software (Becton-Dickinson).

Detection of $m R N A$ expression using real-time PCR. Total RNA of PC-3 was extracted using TRIzol (Invitrogen Life Technologies), according to the manufacturer's instructions. cDNA synthesis was performed with $2 \mu \mathrm{g}$ of total RNA using a RevertAid $^{\mathrm{TM}}$ H Minus First Strand cDNA Synthesis kit (Fermentas). The primers (ProMab) were designed using Primer Express 3.0 software (Applied Biosystems, Carlsbad, CA, USA), and their sequences were: Notch-1, forward: 5'-CCTGTCTGAGGTCAATGAGT-3', reverse: 5'-GTAGCCACTGGTCATGTCTT-3'; Bcl-2, forward: 5'-GAGGATTGTGGCCTTCTT-3', reverse: 5'-CCAGGAGAAATCAAACAGAG-3'; Bax, forward: 5'-CTGAGCAGATCATGAAGACA-3', reverse: 5'-CTCTGCAGCTCCATGTTACT-3' and GAPDH, forward: 5'-CAATGACCCCTTCATTGACC-3', reverse: 5'-GACAAGCTTCCCGTTCTCAG-3'. Quantitative PCR was performed using SYBR-Green Master mix with the use of an ABI PRISM 7500 sequence detection system (Applied 
A

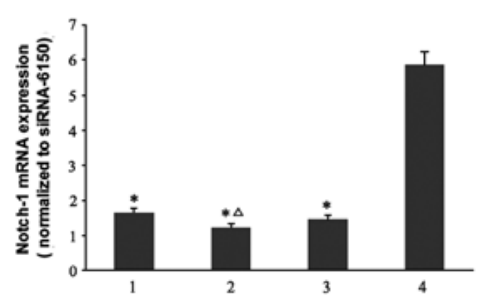

B

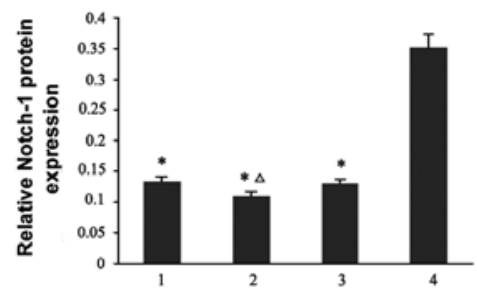

C

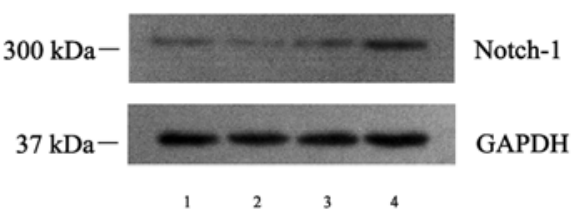

Figure 1. Effects of siRNA-mediated Notch-1 silencing in PC-3 cells. (A) Real-time PCR demonstrates that the expression of Notch-1 mRNA significantly decreases following transfection of three different Notch-1 siRNA fragments. (B) The relative intensity of Notch-1 protein by densitometry. (C) The protein expression level of Notch-1 by western blot analysis. Lane 1, Notch-1 siRNA-2010; lane 2, Notch-1 siRNA-6150; lane 3, Notch-1 siRNA-780; lane 4, scrambled siRNA. ${ }^{*} \mathrm{P}<0.05$, compared with scrambled siRNA; ${ }^{\Delta} \mathrm{P}<0.05$, compared with siRNA-2010 and siRNA-780.

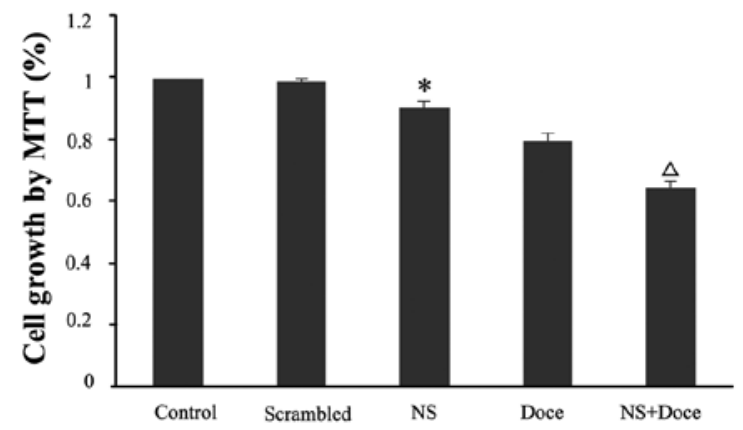

Figure 2. Effects of Notch-1 silencing and docetaxel treatment on the growth of PC-3 cells. MTT assays indicate that cell proliferation in the NS group is significantly inhibited compared to the control and scrambled groups, and cell proliferation in the NS+Doce group is significantly inhibited compared to the NS and Doce groups. ${ }^{*} \mathrm{P}<0.05$, compared with control; ${ }^{\Delta} \mathrm{P}<0.05$, compared with monotherapy.

Biosystems). Amplification data were normalized to the lowest sample, and quantification of the gene expression was performed using the $\Delta \Delta \mathrm{Ct}$ calculation, where $\mathrm{Ct}$ is the threshold cycle; the amount of target gene, normalized to the lowest sample, is given as $2^{-\Delta \Delta \mathrm{Ct}}(17)$.

Detection of protein expression using western blot analysis. The cells were lysed in a lysis buffer at $4^{\circ} \mathrm{C}$ for $30 \mathrm{~min}$. Insoluble material was removed by centrifugation. The protein concentrations were determined using the Bradford protein assay system (Bio-Rad). Protein $(20 \mu \mathrm{g})$ was separated using SDS-PAGE and then transferred to polyvinyl difluoride membranes (Millipore, Bedford, MA, USA) by electroblotting. Following blocking with 5\% non-fat dry milk, the blots were incubated with primary antibodies and developed with alkaline phosphatase-conjugated secondary antibodies (Zymed, San Francisco, CA, USA) using enhanced chemiluminescence (Thermo, Rockford, IL, USA). The membranes were exposed to an X-ray film (Kodak, Rochester, NY, USA). The developed films were subsequently digitized and densitometrically analyzed using Gel-Pro Analyzer software version 4.0 (Media Cybernetics, Silver Spring, MD, USA). The protein expression levels are presented as a densitometric ratio of the targeted protein to the GAPDH housekeeping protein.

Densitometric and statistical analysis. The experiments were performed in triplicate and data are presented as the mean \pm standard deviation. The differences between the sample means were compared using analysis of variance. Analyses were carried out using SPSS for Windows, version 12.1 (SPSS Inc., Chicago, IL, USA). $\mathrm{P}<0.05$ was considered to indicate a statistically significant difference.

\section{Results}

Notch-1 siRNA effectively downregulates Notch-1 expression in PC-3 cells. Three separate Notch-1 siRNA sequences were synthesized. Real-time PCR and western blot analysis were performed $48 \mathrm{~h}$ following transfection to determine siRNA effectiveness. The results demonstrated that Notch-1 mRNA and protein expression were significantly inhibited by the three siRNAs, indicating the efficacy of this approach. Real-time PCR results revealed the inhibition of mRNA expression by the 3 different Notch-siRNA sequences to be 71.9, 79.2 and $74.9 \%$ (Fig. 1A), while western blot analysis revealed the inhibition of Notch-1 receptor protein expression to be 60.9 , 69.2 and $63.2 \%$, respectively (Fig. 1B and C). As the Notch-1 siRNA-6150 had the highest silencing effect, it was used for the subsequent studies.

Downregulation of Notch-1 expression inhibits cell growth and induces apoptosis. To determine whether the Notch pathway is an effective target for treating CRPC, Notch-1 siRNA or scrambled siRNA were transfected into PC-3 cells, and cell survival was determined using an MTT assay $72 \mathrm{~h}$ following transfection. The results demonstrated that cell proliferation in the NS group was significantly inhibited compared to the control and scrambled groups (Fig. 2). To investigate whether the decrease in proliferation was associated with an increased apoptosis, apoptosis was examined using flow cytometry $48 \mathrm{~h}$ following transfection. The results revealed that the apoptosis rates of the control group, scrambled group and Notch-1 siRNA group were 2.56, 2.59 and $8.45 \%$, respectively (Fig. 3). Thus, the Notch-1 siRNA resulted in a 3.3 -fold increase in apoptosis. These results demonstrate that the inhibition of cell proliferation following silencing of the Notch-1 expression is partially due to an increase in apoptosis.

Downregulation of Notch-1 expression increases chemosensitivity. Since the Notch signaling pathway contributes to drug resistance in cancer cells, we examined whether downregulation of Notch-1 sensitized prostate cancer cells to docetaxel, 


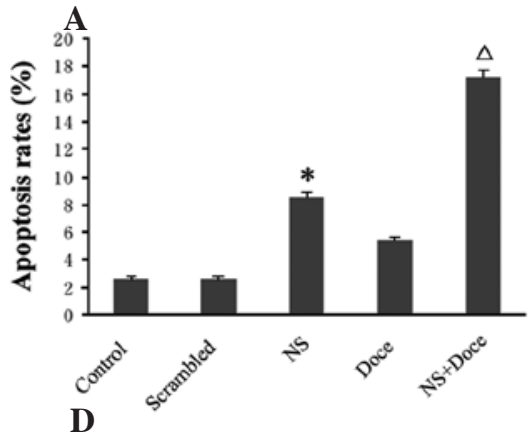

NS

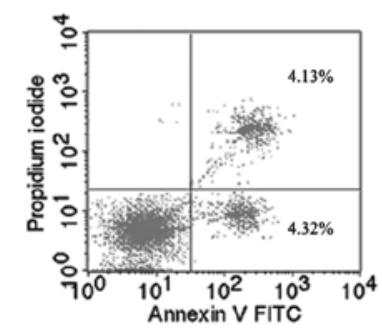

B

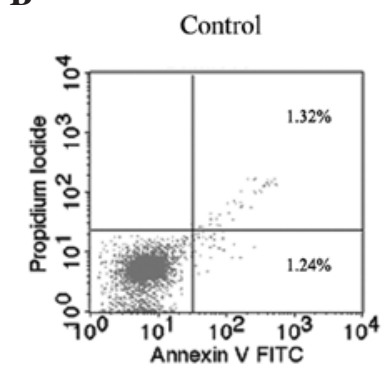

$\mathbf{E}$

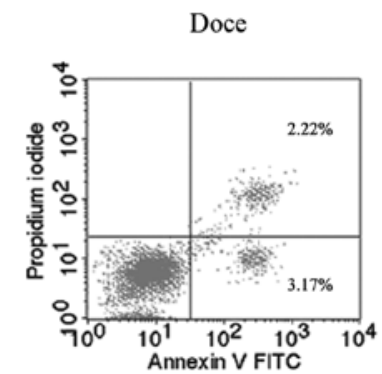

$\mathbf{C}$

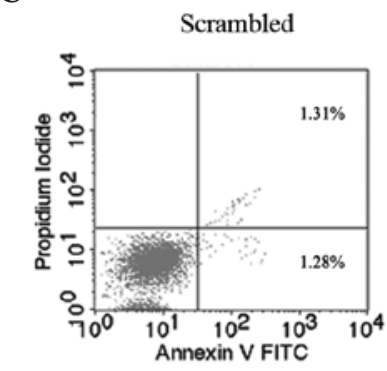

$\mathbf{F}$

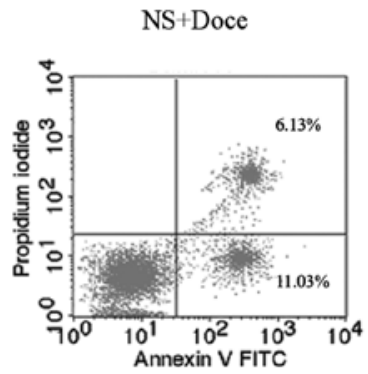

Figure 3. Effects of Notch-1 silencing and docetaxel treatment on apoptosis in PC-3 cells. Apoptosis rates of cells in each group were detected using flow cytometry. The percentage of apoptotic cells in the NS group increased significantly compared to the control and scrambled groups. In addition, the percentage of apoptotic cells increased significantly in the NS+Doce group compared to the Doce group. (A) Columns, mean of at least three experiments. Error bars are the standard deviation. (B-F) Flow charts: lower right quadrant, annexin V-positive and PI-negative cells indicate early apoptotic cells; upper right quadrant, annexin V-and PI-positive cells show necrotic or late apoptotic cells. Early and late apoptotic cells were calculated as the incidence of apoptotic cell death. ${ }^{*} \mathrm{P}<0.05$, compared with control; ${ }^{\Delta} \mathrm{P}<0.05$, compared with monotherapy.

a first-line chemotherapeutic drug for CRPC. A combination of docetaxel and Notch-1 siRNA was used to treat PC-3 cells, and MTT assays were performed to measure the proliferation of the tumor cells. The results revealed that silencing Notch-1 prior to docetaxel treatment significantly potentiated the inhibitory effects of docetaxel on cell proliferation, compared to treatment with docetaxel alone (Fig. 2). To further explore the mechanism underlying the anti-proliferative effect of Notch-1 silencing, we measured apoptosis in tumor cells using flow cytometry. The results revealed that the apoptosis rate following the combination treatment was $17.16 \%$, compared to $5.39 \%$ for docetaxel alone, a 3.2-fold increase (Fig. 3), which suggests that Notch-1 silencing significantly increases the anti-tumor effects of docetaxel.

Effects of Notch-1 gene silencing on the expression of the apoptosis-associated proteins, Bcl-2 and Bax. The Bcl-2 family of proteins are crucial in controlling the apoptotic machinery in mammalian cells via its interacting pro- and anti-apoptotic members. These proteins integrate a wide array of diverse upstream survival and distress signals to determine the fate of the cells (18). Our data indicate that the inhibition of cell proliferation that results from Notch-1 silencing is partially due to an increase in apoptosis. To investigate the mechanism by which Notch-1 silencing regulates apoptosis, we used real-time PCR and western blot analysis to examine the transcriptional activity and protein expression of Bcl-2 and Bax, two Bcl-2 family members known to be significant in the regulation of apoptosis. High levels of Bax and low levels of Bcl-2 expression were observed in the Notch-1 siRNAsilenced cells and docetaxel-treated cells. Furthermore, compared to the siRNA- and docetaxel-alone groups, the expression of $\mathrm{Bcl}-2$ mRNA and protein was significantly downregulated, and the expression of Bax mRNA and protein was significantly upregulated in cells treated with siRNA and docetaxel (Fig. 4).

\section{Discussion}

Notch signaling is significant for the direct cell-cell communication involved in the determination of cell fate, stem cell potential and lineage commitment. The biological function of this pathway is context-dependent (19). For example, upregulation of the Notch gene expression inhibits proliferation and promotes apoptosis in several types of tumor cells, including hepatocellular carcinoma, thyroid cancer and lung adenocarcinoma (20-22). In contrast, in most solid tumors, the Notch gene functions as an oncogene; the occurrence of $\mathrm{PCa}$, kidney cancer, glioma, ovarian cancer and breast cancer are often accompanied by upregulation of the Notch receptor and ligand expression (14,23-28). In PCa, Bin Hafeez et al (14) demonstrated that the expression of Notch-1 is upregulated in pathological specimens and in cell lines PC-3, DU-145, 22Rr1 and LNCaP. Moreover, Notch-1 gene silencing significantly decreases the invasiveness of PC-3 cells (14). In our current results, we found a significant inhibition of PC-3 cell proliferation following Notch-1 silencing, which is associated with increased apoptosis. These findings are consistent with studies by Bin Hafeez et al and Wang et al $(14,15)$, which markedly support a role for Notch-1 as an oncogene in PCa.

As Notch-1 exhibits an anti-tumor function, it has been applied to enhance tumor chemotherapy in in vitro studies. Zang et al demonstrated that silencing Notch-1 significantly increases the sensitivity of breast cancer cells to docetaxel 
A

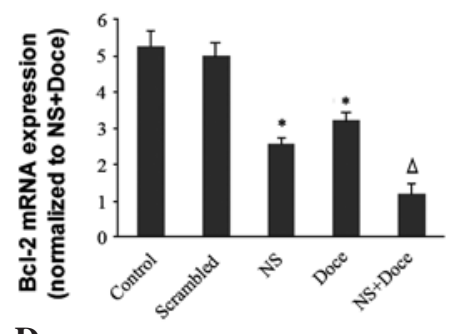

D

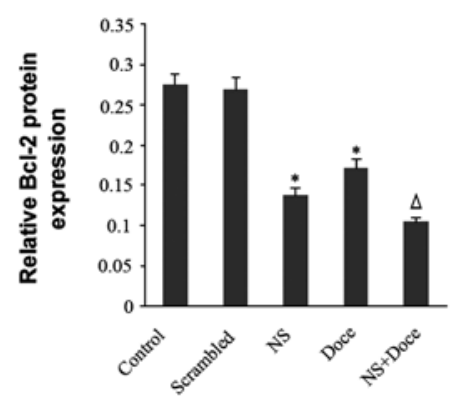

B

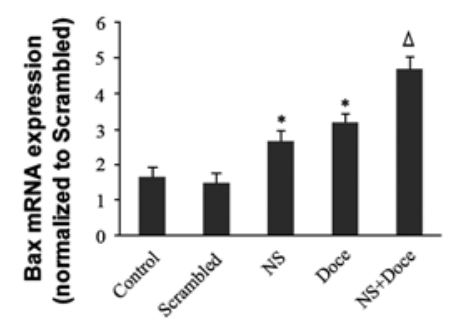

E

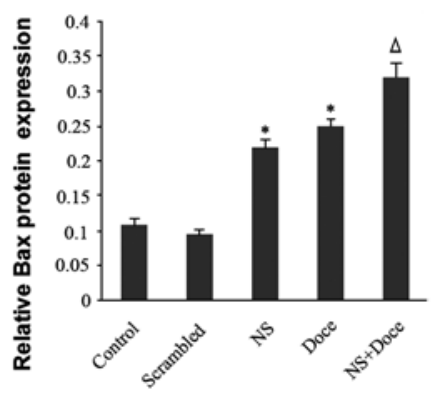

C

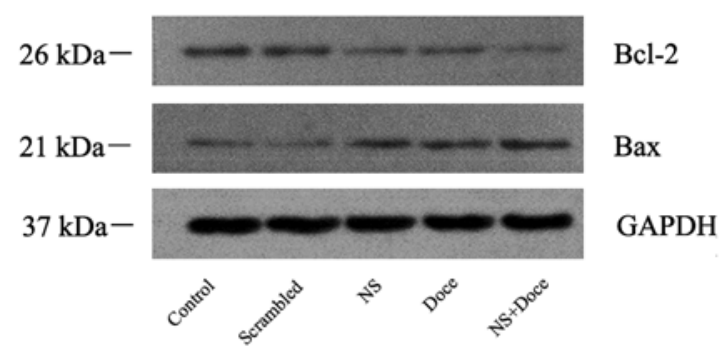

Figure 4. Regulation of Bcl-2 and Bax expression by Notch-1 silencing. (A) The expression of Bcl-2 mRNA is significantly decreased in the NS+Doce group compared to the NS or Doce groups. (B) The expression of Bax mRNA is significantly increased in the NS+Doce group compared to the NS or Doce groups. (C) Bcl-2 protein expression is significantly decreased, and (D) Bax protein expression is significantly increased in the NS+Doce group compared to the NS and Doce groups. (E) The protein expression levels of Bcl-2 and Bax by western blot analysis. ${ }^{~} \mathrm{P}<0.05$, compared with control; ${ }^{\Delta} \mathrm{P}<0.05$, compared with monotherapy.

and doxorubicin (29). The pathogenesis and treatment of $\mathrm{PCa}$ are similar to those of breast cancer; however, its resistance to chemotherapy has been a challenge for clinicians. In our study, siRNA was used to silence the expression of Notch-1 in PC-3 cells, and the synergistic effect between Notch-1 silencing and docetaxel treatment was examined. We determined that the proliferation of the Notch-1-silenced PC-3 cells was significantly inhibited by docetaxel treatment. Furthermore, docetaxel also increased the number of apoptotic PC-3 cells, indicating that the observed decrease in proliferation following combination treatment may arise from the apoptosis-inducing effects of Notch-1. Nefedova et al established a model of multiple myeloma in the mouse and found that inhibition of the Notch signaling pathway by $\gamma$-secretase inhibitors (GSIs) increased the anti-tumor effects of melphalan and doxorubicin (30). Our data indicate that docetaxel chemosensitivity increases in PC-3 cells following silencing of Notch-1 expression by siRNA transfection, an observation that supports the theory that the Notch pathway is an effective target for $\mathrm{PCa}$ treatment.

The ability of taxanes, including paclitaxel and docetaxel, to kill tumor cells depends on their ability to induce mitotic arrest, chromosome segregation failure and apoptosis in tumor cells. The apoptotic signaling pathways in taxane-resistant cell lines have an aberrant expression, which is also one of the reasons for drug resistance in these tumors (31). We demonstrated that the increased docetaxel sensitivity observed in PC-3 cells following Notch-1 silencing could result from the apoptosis-inducing effects of Notch-1. The Notch pathway is mainly involved in cell differentiation; therefore, we explored whether the apoptosisinducing effects of Notch-1 were associated with regulation of the apoptosis signaling pathways. Rasul et al revealed that GSIs induce apoptosis in breast cancer cell lines through the suppression of Bcl-2 and Bcl- $\mathrm{x}_{\mathrm{L}}$ expression (32). Chun and Lee demonstrated that the anti-apoptotic proteins $\mathrm{Bcl}-2$ and $\mathrm{Bcl}-\mathrm{x}_{\mathrm{L}}$ were highly expressed in paclitaxel-resistant hepatoma cell lines, and $\mathrm{Bcl}-\mathrm{x}_{\mathrm{L}}$ expression was enhanced by paclitaxel treatment (33). Bcl-2 inhibits apoptosis, and its overexpression is associated with CRPC. Bak and Bax are members of the Bcl-2 family and counteract the anti-apoptotic functions of Bcl-2 (34). Reagan-Shaw et al studied the effects of vitamin $\mathrm{E}$ and selenium on the LNCaP, DU145 and PC-3 PCa cell lines, and found that these agents induce apoptosis through the upregulation of Bax, Bak and Bid, and the downregulation of Bcl-2 (18). These studies suggest that the anti-tumor effects of chemotherapeutic drugs, including paclitaxel, occur through their regulation of the Bcl-2 signaling pathway. Our results confirm that the high docetaxel sensitivity observed in PC-3 cells was associated with high levels of Bax and low levels of Bcl-2 expression. Although Notch-1 silencing and docetaxel treatment downregulated Bcl-2 and upregulated Bax at the mRNA and protein levels, the effect was more evident when Notch-1 was silenced prior to docetaxel treatment. Therefore, Notch-1 silencing is capable of enhancing the apoptosis-inducing effects of docetaxel, possibly through the regulation of Bcl-2 family members.

In conclusion, our results demonstrate that silencing Notch-1 induces an increase in apoptosis and an inhibition in the proliferation of tumor cells. These results suggest that silencing Notch-1 significantly increases the chemosensitivity of PC-3 cells to docetaxel. Based on these results, it appears that silencing Notch-1 regulates the expression of $\mathrm{Bcl}-2$ family members by downregulating the anti-apoptotic Bcl-2 and upregulating the pro-apoptotic Bax, thus leading to an increased sensitivity of PC-3 cells to docetaxel. This study indicates that Notch-1 is a potential target for the treatment of CRPC. The detailed mechanisms underlying the regulation 
of the Bcl-2 family members by the Notch signaling pathway require further investigation in future studies.

\section{Acknowledgements}

The authors thank Professor Xie Xiao-Ming of the Sun Yat-Sen University Cancer Center (China) for providing the PC-3 cells.

\section{References}

1. American Cancer Society: Cancer Facts and Figures 2011 American Cancer Society, Atlanta, 2011.

2. Eisenberger MA, Blumenstein BA, Crawford ED, et al: Bilateral orchiectomy with or without flutamide for metastatic prostate cancer. N Engl J Med 339: 1036-1042, 1998.

3. Mohler J, Bahnson RR, Boston B, et al: NCCN clinical practice guidelines in oncology: prostate cancer. J Natl Compr Canc Netw 8: 162-200, 2010.

4. Ploussard G, Terry S, Maille P, et al: Class III beta-tubulin expression predicts prostate tumor aggressiveness and patient response to docetaxel-based chemotherapy. Cancer Res 70: 9253-9264, 2010.

5. Petrylak DP, Tangen CM, Hussain MHA, et al: Docetaxel and estramustine compared with mitoxantrone and prednisone for advanced refractory prostate cancer. New Engl J Med 351: 1513-1520, 2004.

6. Artavanis-Tsakonas S, Rand MD and Lake RJ: Notch signaling: cell fate control and signal integration in development Science 284: 770-776, 1999.

7. Kopan R and Ilagan MX: The canonical Notch signaling pathway: unfolding the activation mechanism. Cell 137: 216-233, 2009

8. Miele L: Notch signaling. Clin Cancer Res 12: 1074-1079, 2006.

9. Miele L, Golde T and Osborne B: Notch signaling in cancer. Curr Mol Med 6: 905-918, 2006.

10. Leong KG and Gao WQ: The Notch pathway in prostate development and cancer. Differentiation 76: 699-716, 2008.

11. Villaronga MA, Bevan CL and Belandia B: Notch Signaling: a potential therapeutic target in prostate cancer. Curr Cancer Drug Tar 8: 566-580, 2008.

12. Shou JY, Ross S, Koeppen H, de Sauvage FJ and Gao WQ: Dynamics of notch expression during murine prostate development and tumorigenesis. Cancer Res 61: 7291-7297, 2001.

13. Scorey N, Fraser SP, Patel P, Pridgeon C, Dallman MJ and Djamgoz MBA: Notch signalling and voltage-gated $\mathrm{Na}^{+}$channel activity in human prostate cancer cells: independent modulation of in vitro motility. Prostate Cancer Prostatic Dis 9: 399-406, 2006.

14. Bin Hafeez B, Adhami VM, Asim M, et al: Targeted knockdown of Notch1 inhibits invasion of human prostate cancer cells concomitant with inhibition of matrix metalloproteinase-9 and urokinase plasminogen activator. Clin Cancer Res 15: 452-459, 2009.

15. Wang ZW, Li Y, Banerjee S, et al: Down-regulation of Notch-1 and Jagged-1 inhibits prostate cancer cell growth, migration and invasion, and induces apoptosis via inactivation of Akt, mTOR, and NF-kappa B signaling pathways. J Cell Biochem 109: 726-736, 2010

16. Bushman W, Gipp J, Gu GY, Crylen C and Kasper S: Hedgehog pathway activity in the LADY prostate tumor model. Mol Cancer 6: 19, 2007.
17. Livak KJ and Schmittgen TD: Analysis of relative gene expression data using real-time quantitative PCR and the 2(-Delta Delta C(T)) method. Methods 25: 402-408, 2001.

18. Reagan-Shaw S, Nihal M, Ahsan H, Mukhtar H and Ahmad N: Combination of vitamin $\mathrm{E}$ and selenium causes an induction of apoptosis of human prostate cancer cells by enhancing Bax/Bcl-2 ratio. Prostate 68: 1624-1634, 2008.

19. Dotto GP: Notch tumor suppressor function. Oncogene 27: 5115-5123, 2008

20. Wang M, Xue L, Cao Q, et al: Expression of Notch1, Jagged1 and beta-catenin and their clinicopathological significance in hepatocellular carcinoma. Neoplasma 56: 533-541, 2009.

21. Kunnimalaiyaan M, Vaccaro AM, Ndiaye MA and Chen $\mathrm{H}$ : Overexpression of the NOTCH1 intracellular domain inhibits cell proliferation and alters the neuroendocrine phenotype of medullary thyroid cancer cells. J Biol Chem 281: 39819-39830, 2006.

22. Zheng Q, Qin $\mathrm{H}$, Zhang $\mathrm{H}$, et al: Notch signaling inhibits growth of the human lung adenocarcinoma cell line A549. Oncol Rep 17: 847-852, 2007

23. Miyamoto Y, Maitra A, Ghosh B, et al: Notch mediates TGF alpha-induced changes in epithelial differentiation during pancreatic tumorigenesis. Cancer Cell 3: 565-576, 2003.

24. Zagouras P, Stifani S, Blaumueller CM, Carcangiu ML and Artavanis-Tsakonas S: Alterations in Notch signaling in neoplastic lesions of the human cervix. Proc Natl Acad Sci USA 92: 6414-6418, 1995.

25. Rae FK, Stephenson SA, Nicol DL and Clements JA: Novel association of a diverse range of genes with renal cell carcinoma as identified by differential display. Int J Cancer 88: 726-732, 2000.

26. Purow BW, Haque RM, Noel MW, et al: Expression of Notch-1 and its ligands, Delta-like-1 and Jagged-1, is critical for glioma cell survival and proliferation. Cancer Res 65: 2353-2363, 2005.

27. Hopfer O, Zwahlen D, Fey MF and Aebi S: The Notch pathway in ovarian carcinomas and adenomas. Br J Cancer 93: 709-718, 2005.

28. Reedijk M, Odorcic S, Chang L, et al: High-level coexpression of JAG1 and NOTCH1 is observed in human breast cancer and is associated with poor overall survival. Cancer Res 65: 8530-8537, 2005.

29. Zang SL, Chen F, Dai JJ, et al: RNAi-mediated knockdown of Notch-1 leads to cell growth inhibition and enhanced chemosensitivity in human breast cancer. Oncol Rep 23: 893-899, 2010

30. Nefedova Y, Sullivan DM, Bolick SC, Dalton WS and Gabrilovich DI: Inhibition of Notch signaling induces apoptosis of myeloma cells and enhances sensitivity to chemotherapy. Blood 111: 2220-2229, 2008.

31. McGrogan BT, Gilmartin B, Camey DN and McCann A: Taxanes, microtubules and chemoresistant breast cancer. Biochim Biophys Acta 1785: 96-132, 2008.

32. Rasul S, Balasubramanian R, Filipovic A, Slade MJ, Yague E and Coombes RC: Inhibition of gamma-secretase induces $\mathrm{G} 2 / \mathrm{M}$ arrest and triggers apoptosis in breast cancer cells. Br J Cancer 100: 1879-1888, 2009.

33. Chun $\mathrm{E}$ and Lee KY: Bcl-2 and Bcl- $\mathrm{X}_{\mathrm{L}}$ are important for the induction of paclitaxel resistance in human hepatocellular carcinoma cells. Biochem Biophys Res Commun 315: 771-779, 2004.

34. Yoshino T, Shiina H, Urakami S, et al: Bcl-2 expression as a predictive marker of hormone-refractory prostate cancer treated with taxane-based chemotherapy. Clin Cancer Res 12: 6116-6124, 2006. 